\title{
Current Single Event Effects Results for Candidate Spacecraft Electronics for NASA
}

\author{
Martha V. O'Bryan, Christina M. Seidleck, \\ Martin A. Carts \\ Raytheon/ITSS \\ NASA/GSFC Code 561.4 \\ Greenbelt, MD USA \\ matha.obryan@gsfc.nasa.gov
}

\author{
Kenneth A. LaBel, Cheryl J. Marshall, \\ Robert A. Reed, Anthony B. Sanders, \\ Donald K. Hawkins, Stephen R. Cox \\ NASA/GSFC Code 561.4 \\ Greenbelt, MD USA \\ Kenneth.A.Label@nasa.gov
}

\author{
Scott D. Kniffin, Ray L. Ladbury, \\ Mark Walter, Christopher Palor \\ Orbital Sciences Corporation \\ McLean, VA USA \\ Scott.D.Kniffin.1@gsfc.nasa.gov
}

Moses McCall, Steve Meyer, Elionex Rodriguez, Dave Rapchun

NASA/GSFC Code 565 (Rapchun 685)

Greenbelt, MD USA

\author{
James W. Howard, Jr., Hak S. Kim, \\ James D. Forney \\ Washington, DC USA \\ James.W.Howard.1@gsfc.nasa.gov
}

Stephen P. Buchner, Timothy R. Oldham, John Sutton, Timothy L Irwin

QSS Group, Inc.

Greenbelt, MD USA

\author{
Dale McMorrow \\ Naval Research Laboratory \\ Washington, DC USA
}

Paul W. Marshall

Consultant

Brookneal, VA USA

John Lintz

Honeywell Defense and Space Electronic Systems Clearwater, FL USA

\author{
John Rodgers, Suhail Mohammed \\ Motorola Libraries and Memories \\ Austin, TX USA
}

\begin{abstract}
We present data on the vulnerability of a variety of candidate spacecraft electronics to proton and heavy ion induced single event effects. Devices tested include digital, analog, linear bipolar, and hybrid devices, among others.
\end{abstract}

Keywords-component; SEE, add others

\section{INTRODUCTION}

As spacecraft designers use increasing numbers of commercial and emerging technology devices to meet stringent performance, as well as economic and schedule requirements, ground-based testing of such devices for susceptibility to single event effects (SEE) has assumed ever greater importance. The studies discussed here were undertaken to establish the sensitivities of candidate spacecraft electronics to heavy ion and proton-induced single event upsets (SEU), single event

The Authors would like to acknowledge the sponsors of this effort: NASA Electronic Parts and Packaging Program (NEPP), NASA Flight Projects, and the Defense Threat Reduction Agency (DTRA) under IACRO 03-40351 and 04-40641. latchup (SEL), and single event transient (SET). Note: For proton displacement damage (DD) and total ionizing dose (TID) results please see the companion poster W-5 entitled "Current Total Ionizing Dose and Displacement Damage Results for Candidate Spacecraft Electronics for NASA" by Donna Cochran, et al. that is also being presented at the IEEE Nuclear and Space Radiation Effects Conference (NSREC) Data Workshop [1].

\section{Test TeChNiques AND SETUP}

\section{A. Test Facilities}

All SEE tests were performed between February 2003 and February 2004. Heavy ion experiments were conducted at the Texas A\&M University (TAMU) Cyclotron [2] and Singleevent effects facility (SEETF) at the National Superconducting Cyclotron Laboratory (NSCL) at Michigan State University (MSU)[3]. The SEUTF uses a twin Tandem Van de Graaff

Presented by Martha O'Bryan at NSREC04 Data Workshop, Atlanta, GA, July 22, 2004 
accelerator while the TAMU facility uses an 88 " cyclotron. The NSCL uses tandem K500 and K1200 cyclotrons to deliver on target ions with energies up to $125 \mathrm{MeV} / \mathrm{n}$. Both facilities provide a variety of ions over a range of energies for testing. At both facilities, test boards containing the device under test (DUT) were mounted in the test area. For heavy ions, the DUT was irradiated with ions with linear energy transfers (LETs) ranging from 0.59 to $174 \mathrm{MeV} \cdot \mathrm{cm}^{2} / \mathrm{mg}$. Fluxes ranged from $7.7 \times 10^{1}$ to $2.5 \times 10^{5}$ particles $/ \mathrm{cm}^{2}$ per second, depending on the device sensitivity. Representative ions used are listed in Table I. LETs between the values listed were obtained by changing the angle of incidence of the ion beam on the DUT, thus changing the path length of the ion through the DUT and the "effective LET" of the ion. Energies and LETs available varied slightly from one test date to another.

Proton SEE tests were performed at two facilities: the University of California at Davis (UCD) Crocker Nuclear Laboratory (CNL) [4], and the Indiana University Cyclotron Facility (IUCF) [5]. Proton test energies incident on the DUT are listed in Table II. Proton SEE tests were performed in a manner similar to heavy ion exposures. However, because protons cause SEE via indirect ionization of recoil particles, results are parameterized in terms of proton energy rather than LET. Proton tests also feature higher cumulative fluence and particle flux rates than do heavy-ion experiments.

Laser SEE tests were performed at the pulsed laser facility at the Naval Research Laboratory (NRL) [6] [7]. The laser light had a wavelength of $590 \mathrm{~nm}$ resulting in a skin depth (depth at which the light intensity decreased to $1 / \mathrm{e}$ - or about $37 \%$ - of its intensity at the surface) of $2 \mathrm{~mm}$. A pulse rate of $100 \mathrm{~Hz}$ was chosen.

TABLE I. HEAVY ION TEST FACTLITIES AND TEST HEAVY IONS

\begin{tabular}{|c|c|c|c|c|}
\hline & Ion & $\begin{array}{c}\text { Energy, } \\
\mathrm{MeV}\end{array}$ & $\begin{array}{c}\text { LET in Si, } \\
\text { MeV.cm²/mg }\end{array}$ & $\begin{array}{c}\text { Normal } \\
\text { Incidence } \\
\text { Range in } \\
\mathrm{Si}, \mu \mathrm{m}\end{array}$ \\
\hline \multirow{12}{*}{ TAMU } & $\mathrm{Ne}^{20}$ & $264-285$ & $2.5-2.81$ & $262-331$ \\
\hline & $\mathrm{Ar}^{40}$ & $496-561$ & $8.05-8.9$ & $174-224$ \\
\hline & $\mathrm{Cu}^{63}$ & 750 & 19.95 & 120 \\
\hline & $\mathrm{Kr}^{\mathrm{Bd}}$ & $912-953$ & $28-29.3$ & $116-122$ \\
\hline & $\mathrm{Ag}^{107}$ & 1200 & 42.85 & 100 \\
\hline & $x e^{129}$ & $1291-1722$ & $49.3-54$ & $102-127$ \\
\hline & $\mathrm{Au}^{197}$ & 2955 & 80.2 & 155 \\
\hline & $-\mathrm{Ne}^{20}$ & 545 & 1.7 & 799 \\
\hline & $A r^{40}$ & 991 & 5.4 & 493 \\
\hline & $\mathrm{Kr}^{84}$ & 2081 & 19.3 & 332 \\
\hline & $x e^{129}$ & 3197 & 37.9 & 286 \\
\hline & \multicolumn{4}{|c|}{ - $25 \mathrm{MeV}$ per nucleon tune } \\
\hline \multirow{4}{*}{ MSU } & $\mathrm{Ar}^{36}$ & 5148 & 1.5 & 8860 \\
\hline & $\mathrm{Kr}^{78}$ & 9438 & 6.8 & 4440 \\
\hline & $X e^{136}$ & 17816 & 14.1 & 3070 \\
\hline & $\mathrm{Bi}^{209}$ & 15048 & 42 & 1100 \\
\hline
\end{tabular}

\section{TABLE II. PROTON TEST FACHITIES AND PARTICLES}

\begin{tabular}{|l|c|c|}
\hline \multicolumn{1}{|c|}{ Facility } & Particle & $\begin{array}{c}\text { Particle } \\
\text { Energy,(MeV) }\end{array}$ \\
\hline $\begin{array}{l}\text { University of Califomia at Davis (UCD) } \\
\text { Crocker Nuclear Laboratory (CNL) }\end{array}$ & Proton & $26.6-63$ \\
\hline Indiana University Cyclotron Facility (IUCF) & Proton & $54-197$ \\
\hline
\end{tabular}

TABLE III. OTHER TEST FACIITIES

Naval Research Laboratory (NRL) Pulsed Laser SEE Test Facility

Laser: $590 \mathrm{~nm}, 1$ ps pulse width, beam spot size $-1.2 \mu \mathrm{m}$

\section{B. Test Method}

Unless otherwise noted, all tests were performed at room temperature and with nominal power supply voltages.

\section{1) SEE Testing - Heavy Ion}

Depending on the DUT and the test objectives, one or more of three SEE test methods were used:

Dynamic - the DUT was exercised contimually while being exposed to the beam. The errors were counted, generally by comparing DUT output to an unirradiated reference device or other expected output. In some cases, the effects of clock speed or device modes were investigated. Results of such tests should be applied with caution because device modes and clock speed can affect SEE results.

Static - the DUT was loaded prior to irradiation; data were retrieved and errors were counted after irradiation.

Biased (SEL only) - the DUT was biased and clocked while $I_{O C}$ (power consumption) was monitored for SEL or other destructive effects. In some SEL tests, functionality was also monitored.

In SEE experiments, DUTs were monitored for soft errors, such as SEUs and for hard errors, such as SEL. Detailed descriptions of the types of errors observed are noted in the individual test results.

SET testing was performed using a high-speed oscilloscope. Individual criteria for SETs are specific to the device being tested. Please see the individual test reports for details. [9]

Heavy ion SEE sensitivity experiments include measurement of the saturation cross sections and the Linear Energy Transfer (LETth) threshold (the maximum LET value at which no effect was observed at an effect fluence of $1 \times 10^{7}$ particles $/ \mathrm{cm}^{2}$ ).

\section{2) SEE Testing - Proton}

Proton SEE tests were performed in a manner similar to heavy ion exposures in many regards. Differences include measuring the SEE cross section as a function of proton energy as opposed to LET, as well as differences in cumulative fluence and particle flux rates.

Presented by Martha O'Bryan at NSREC04 Data Workshop, Atlanta, GA, July 22, 2004 


\section{3) Pulsed Laser Facility Testing}

The DUT was mounted on an X-Y-Z stage in front of a $100 \mathrm{x}$ lens that produced a spot size of about $1.2 \mu \mathrm{m}$ FWHM. The X-Y-Z stage could be moved in steps of $0.1 \mu \mathrm{m}$ for accurate positioning of SEU sensitive regions in front of the focused beam. An illuminator together with a CCD camera and monitor were used to image the area of interest, thereby facilitating accurate positioning of the device in the beam. The pulse energy was varied in a continuous manner using a polarizer/half-waveplate combination and the energy was monitored by splitting off a portion of the beam and directing it at a calibrated energy meter.

\section{4) Charge Collection Testing}

A four probe Ion Beam Induced Charge Collection (IBICC) measurement was used to simultaneously measure the charge presented on the collector, emitter, base, and substrate terminal due to a series of ion strikes occurring in and around the transistor's area.

\section{Test Results OVERVIEW}

Abbreviations and conventions are listed in Table IV. Abbreviations for principal investigators (PIs) are listed in Table V. SEE test result categories are summarized in Table VI and SEE results are summarized in Table VII. Unless otherwise noted, all LETs are in $\mathrm{MeV} \cdot \mathrm{cm}^{2} / \mathrm{mg}$ and all cross sections are in $\mathrm{cm}^{2} /$ device. This paper is a summary of results. Complete test reports are available online at http://radhome.gsfc.nasa.gov [8].

TABLE N. ABBREVIATIONS AND CONVENTIONS
TABLE V. LIST OF PRINCIPAL INVESTIGATORS

\begin{tabular}{|c|l|}
\hline Abbreviation & Principal Investigator (PI) \\
\hline SB & Steve Buchner \\
\hline JH & Jim Howard \\
\hline SK & Scott Kniffin \\
\hline RL & Ray Ladbury \\
\hline PM & Paul Marshall \\
\hline TO & Tim Oldham \\
\hline RR & Robert Reed \\
\hline TS & Anthony (Tony) Sanders \\
\hline
\end{tabular}

\section{TABLE VI. LIST OF CATEGORIES}

Following ground SEE irradiation, devices generally are categorized into "useability" categories for spacecraft interest. Recommendations for SEE are color coded according to the following key:

\begin{tabular}{|ll|}
\hline Category 1: & $\begin{array}{l}\text { Recommended for usage in all NASAGSFC } \\
\text { spaceflight applications }\end{array}$ \\
\hline Category 2: & $\begin{array}{l}\text { Recommended for usage in NASAGSFC spaceflight } \\
\text { applications, but may require mitigation techniques }\end{array}$ \\
\hline Category 3: & $\begin{array}{l}\text { Recommended for usage in some NASAGSFC spaceflight } \\
\text { applications, but requires extensive mitigation tochniques or } \\
\text { hard failure recovery mode }\end{array}$ \\
\hline Category 4: & $\begin{array}{l}\text { Not recommended for usage in any NASAGSFC } \\
\text { spaceflight applications }\end{array}$ \\
\hline RTV: & $\begin{array}{l}\text { Research Test Vehicte - Please contact the P.I. before } \\
\text { utilizing this device for spacellight applications }\end{array}$ \\
\hline
\end{tabular}

$H=$ heavy ion test

$P=$ proton test (SEE)

$L=$ laser test

$\mathrm{CC}=$ Charge Collection

LET = linear energy transfer ( $\mathrm{MeV} \cdot \mathrm{cm}^{2} / \mathrm{mg}$ )

LETth = linear energy transfer threshold (the maximum LET value at which no effect was observed at an effective fluence of $1 \times 10^{7}$ particles/cm2)

SEE = single event effects

SEU = single event upset

SEL = single event latchup

SET $=$ single event transient

SEFI = single event functional interrupt

SEB = single event burnout

SEGR = single event gate rupture

$\mathrm{BER}=$ Bit Error Rate
$<$ = SEE observed at lowest tested LET

$>=$ No SEE observed at highest tested LET

$\sigma=$ cross section $\left(\mathrm{cm}^{2} /\right.$ device, unless specified as $\mathrm{cm}^{2} / \mathrm{bit}$ )

$\sigma_{\mathrm{SAT}}=$ saturation cross section at $L E T_{\text {MAX }}$ ( $\mathrm{cm}^{2} /$ device, unless specified as $\left.\mathrm{cm}^{2} / \mathrm{bit}\right)$

LDC $=$ Lot Date Code

$\mathrm{DAC}=$ Digital to Analog Converter

DUT $=$ Device Under Test

N/A $=$ Not Applicable

Cat. = Category

P.I. = Principal Investigator

Samp. = Sample

$H I=$ Heavy lon

$P=$ Proton

NA $=$ Not Applicable

Cat. $=$ Category
ADC = Analog to Digital Converter

ASIC = Application Specific Integrated Circuit

BERT = Bit Error Rate Tester

CMOS = Complementary Metal Oxide Semiconductor

DAC = Digital to Analog Converter

FPGA $=$ Field Programmable Gate Array

HBT = Heterojunction Bipolar Transistor

NV = Non-Volatile

NVM $=$ Non-Volatile Memory

OPTO = Optocoupler

PWM = Pulse Width Modulator

RHrFPGA = Radiation Hardened Reprogrammable Fieldprogrammable Gate Array 
TABLE VII. SUMMARY OF SEE TEST RESULTS

\begin{tabular}{|c|c|c|c|c|c|c|c|c|c|c|}
\hline $\begin{array}{c}\text { Part } \\
\text { Number }\end{array}$ & Manufacturer & $\angle D C$ & $\begin{array}{l}\text { Device } \\
\text { Function }\end{array}$ & Process & $\begin{array}{l}\text { Particle: } \\
\text { (Facily,Date) } \\
\text { P.I. }\end{array}$ & 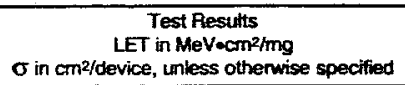 & $\begin{array}{l}\text { SEE } \\
\text { Cat. }\end{array}$ & $\begin{array}{l}\text { Supply } \\
\text { Voltage }\end{array}$ & \begin{tabular}{|} 
Samp. \\
Size
\end{tabular} & Test Report \\
\hline
\end{tabular}

Logic Devices

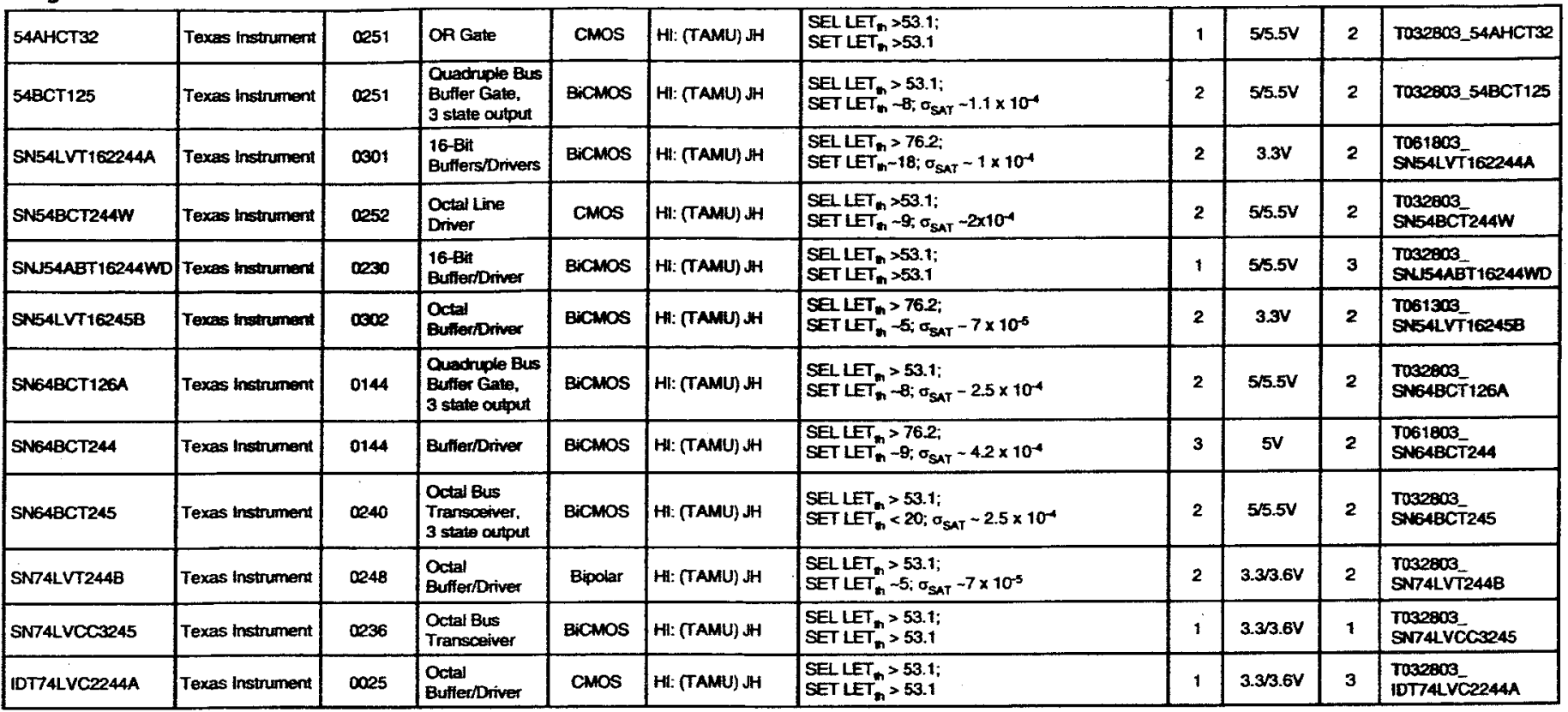

Menory Devices

\begin{tabular}{|c|c|c|c|c|c|c|c|c|c|c|}
\hline $1+x \leqslant 228$ & Honeymel & $\left|\begin{array}{c}N o L D C \\
\text { (Test Crip) }\end{array}\right|$ & $128 k \times 8$ SRAM & sor & $\begin{array}{l}\text { P: (IU) SK } \\
\text { P: (UCD) SK }\end{array}$ & $\begin{array}{l}\text { IU: SET } \sigma_{\max } 2.1 \times 10^{-11}(\sigma \text { varies with angle) } \\
\text { UCD: SET } \sigma_{\max } 2.1 \times 10^{-11}(a \text { varios with angle) }\end{array}$ & 2 & $5 v$ & 2 & D031504_RS422 \\
\hline CPAM test chip & $\begin{array}{l}\text { BAE Systems / } \\
\text { Ovonyx }\end{array}$ & $\left|\begin{array}{c}\text { No LDC } \\
\text { (Test Chip) }\end{array}\right|$ & $\begin{array}{l}\text { Chaloogyenide } \\
\text { PẠM }\end{array}$ & CMOS & H: (TAMU) TO & 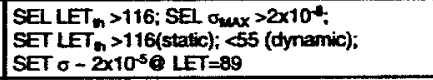 & 2 & $3.3 \mathrm{~V}$ & 2 & TOQ91603_CRAM \\
\hline Floating Gate & Motorola & $\begin{array}{c}\text { No LDC } \\
\text { (Test Chip) }\end{array}$ & \begin{tabular}{|l}
$512 \mathrm{k} \times \mathrm{B}$ \\
NV Memory \\
\end{tabular} & CMOS & H: (TAMU) TO & $\begin{array}{l}\text { SEL LET } \\
\text { SEU }>53 \text { : } \\
\text { SET }\end{array}$ & 2 & $5.5 / 4 \mathrm{~V}$ & 1 & T022204_Motorola \\
\hline Si-Nano NMMS & Motorola & $\mid \begin{array}{c}\text { No } L D C \\
\text { (Test Chip) }\end{array}$ & $\begin{array}{l}512 \mathrm{k} \times \mathrm{B} \\
\mathrm{NV} \text { Mernory }\end{array}$ & $\begin{array}{c}\text { CMOS } \\
\text { rano-cystal }\end{array}$ & H:(TAMU) TO & $\begin{array}{l}\text { SEL LET }{ }_{n}>53: \\
\text { SEU LET } \\
\text { SEU } \sigma>2 \times 10^{-1} \text { : LET }=53\end{array}$ & 2 & $3.3 / 2.2 \mathrm{~V}$ & 5 & T022204_Motorola \\
\hline M65656 & Matra & 9535 & $32 \mathrm{k} \times 8 \mathrm{SFAM}$ & CMOS & HI: (MSU) RL/AR & $\begin{array}{l}\text { SEU } \sigma=1.25 \times 10^{-2} \text { LET }=6.3 \\
\text { SEU } \sigma=4.9 \times 10^{-2} \text { LET }=8.7\end{array}$ & 2 & sv & 1 & $\begin{array}{l}\text { ret: Late news MSU } \\
\text { put by RRRRL }\end{array}$ \\
\hline IDT71256 & IDT & N/A & 256k Memory & CMOS & H: (MSU) RL/RA & $\begin{array}{l}\text { SEU } \sigma=1.0 \times 10^{-3} \text { LET }=6.3 \\
\text { SEU } \sigma=4.5 \times 10^{-3} \cup \text { LET }=8.7\end{array}$ & 2 & $5 \mathrm{v}$ & 1 & $\begin{array}{l}\text { ret. Late news MSU } \\
\text { pub by RRARL }\end{array}$ \\
\hline
\end{tabular}

ASIC Devices

\begin{tabular}{|c|c|c|c|c|c|c|c|c|c|c|}
\hline T36T-GAFE7 & Agilent & Nove3 & ASIC & CMOS & HI: (TAMU) SK & $\begin{array}{l}2.7<S E U L_{L T}<8.3, \text { SEU } \sigma_{\text {SAT }}-4 \times 10^{-5} \\
\text { SEL LET } \\
\text { m }>51.5\end{array}$ & 3 & $5 V$ & 2 & TI11103_GLAST \\
\hline T36T-GAFE5G & Agilent & Sep03 & ASIC & CMOS & H: (TAMU) SK. & 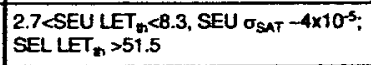 & 3 & $5 \mathrm{v}$ & 2 & T111103_GLAST \\
\hline T36T-GARCB & Agilent & NowO3 & Asic & CMOS & H: (TAMU) SK & 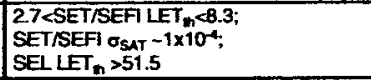 & $\mathbf{3}$ & $5 \mathrm{~V}$ & 2 & T111103_GLAST \\
\hline
\end{tabular}

Mixed Signal or Linear Devices

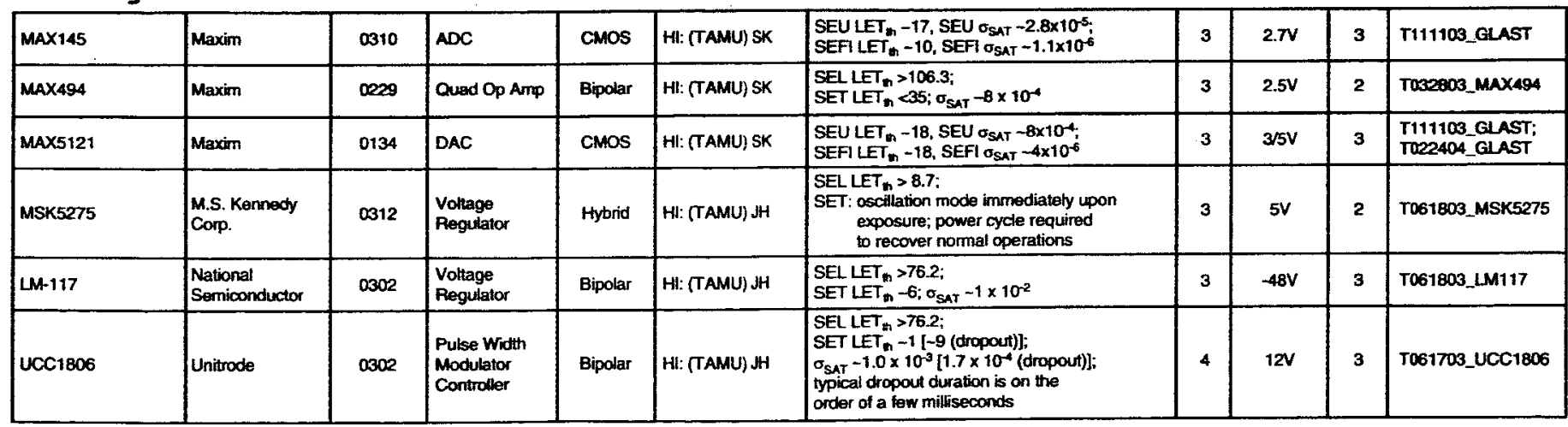

Presented by Martha O'Bryan at NSREC04 Data Workshop, Atlanta, GA, July 22, 2004

Page 4 of 8 
TABLE VII. SUMMARY OF SEE TEST RESULTS (CONT.)

\begin{tabular}{|c|c|c|c|c|c|c|c|c|c|c|}
\hline $\begin{array}{c}\text { Part } \\
\text { Number }\end{array}$ & Manutacturer & LDC & $\begin{array}{l}\text { Device } \\
\text { Function }\end{array}$ & Process & $\begin{array}{l}\text { Particle: } \\
\text { (Facility,Date) } \\
\text { P.I. }\end{array}$ & $\begin{array}{c}\text { Test Results } \\
\text { LFT in MeVtan'/mg } \\
\sigma \text { in cm?/device, unless oftherwise specified }\end{array}$ & $\begin{array}{l}\text { SEE } \\
\text { Cat }\end{array}$ & $\begin{array}{l}\text { Supply } \\
\text { Voltage }\end{array}$ & $\begin{array}{c}\text { Samp. } \\
\text { Size }\end{array}$ & Test Preport \\
\hline
\end{tabular}

DC-DC Converters and Related Devices

\begin{tabular}{|c|c|c|c|c|c|c|c|c|c|c|}
\hline DVFL286R7S-A & VPT & $\begin{array}{c}\text { F0001 \& } \\
\text { F0004 }\end{array}$ & $\begin{array}{l}\text { DCDC } \\
\text { Converter }\end{array}$ & Hybrid & HI: (TAMU) SB/JH & $\begin{array}{l}\text { SEL LET } \\
\text { SET LET }>53.1 \text {; } \\
\text { input voltage and output load conditions]; } \\
\text { worst case condition } 28 \mathrm{~V} \text { input } \\
90 \% \text { load } 7 \times 10^{-5}\end{array}$ & 3 & $28 \mathrm{~V}$ & 2 & T032703_DV \\
\hline DVTR28150-R & VPT & $\begin{array}{c}F 000028 \\
F 0004\end{array}$ & $\begin{array}{l}\text { DCrOC } \\
\text { Converter }\end{array}$ & Hybrid & H: (TAMU) SEVJH & $\begin{array}{l}\text { SEL LET } \\
\text { SET } 2 \text { LET } \\
\text { n }-28.1 \text {; }\end{array}$ & 2 & $28 \mathrm{~V}$ & 2 & T032703_DV \\
\hline $\operatorname{MAX1523}$ & Maxim & $\begin{array}{l}\text { Martings: } \\
\text { AAOY CYR }\end{array}$ & $\begin{array}{l}\text { DCroc boost } \\
\text { Controller }\end{array}$ & cMos & HI: (TAMU) SK & $\begin{array}{l}\text { SET and SEL LET }>76.2 \\
\text { SET and SEL } \sigma<1 \times 10^{-7} Q \quad \angle E T=762\end{array}$ & 1 & $5 \mathbf{V}$ & 2 & T061703_GLAST \\
\hline $\max 1809$ & Maxim & 0301 & $\begin{array}{l}\text { DCDC } \\
\text { Comvater }\end{array}$ & camos & H: (TAMU) SK & SET LET & 3 & $5 V$ & 2 & T061703_GLAST \\
\hline$M \times 1847$ & Maxim & 0313 & $\begin{array}{l}\text { Invarting } \\
\text { DCADC Comerter }\end{array}$ & cmos & HH: (TAMM) SK & 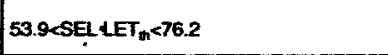 & 4 & SV & 2 & TOG1703_GLAST \\
\hline MAX1951 & Maxim & 0237 & $\begin{array}{l}\text { DCACC } \\
\text { Regutator }\end{array}$ & cros & HI: (TAMU) SK & $\begin{array}{l}\text { 2.8<SET 1ET } \\
\text { SET } \sigma=1.7 \times 10^{-4}\end{array}$ & 4 & $5 V$ & 2 & TOGTT03_GLAST \\
\hline $\operatorname{max724}$ & Maxim & 0248 & $\begin{array}{l}\text { DCDC } \\
\text { Regulator }\end{array}$ & Bipolar & HI: (TAMU) SK & $\begin{array}{l}\text { SEL and SET LET }>>76.2 ; \\
\text { SET and SEL } \sigma<1 \times 10^{8}\end{array}$ & 1 & $5 V$ & 4 & T061703_GLAST \\
\hline $\operatorname{MA} \times 726$ & Maxim & 0313 & $\begin{array}{l}\text { DC/DC } \\
\text { Pegulator }\end{array}$ & Bipolar & HI: (TAMU) SK & $\begin{array}{l}\text { SEL and SET LET } \\
\text { SET and SEL } \sigma<1 \times 16.2 \text {; }\end{array}$ & 1 & 5V & 3 & $\begin{array}{l}\text { T111103_GLAST; } \\
\text { T061703_GLAST }\end{array}$ \\
\hline AFL2803P3S & $\begin{array}{l}\text { International } \\
\text { Rectifier }\end{array}$ & 0351 & $\begin{array}{l}\text { DCDC } \\
\text { Converter }\end{array}$ & Hybrid & HI: (TAMU) SK & 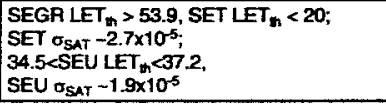 & 3 & $27 / 28 / 29 \mathrm{~V}$ & 2 & $\begin{array}{l}\text { T022304_MAX724 } \\
\text { AFL2803735 }\end{array}$ \\
\hline
\end{tabular}

SiGe Devices

\begin{tabular}{|c|c|c|c|c|c|c|c|c|c|c|}
\hline 5AM [Test Sample] & IBM & NA & $\begin{array}{l}\text { SiGe } \\
\text { 5AMSTC }\end{array}$ & SAM & H: (TAMU) PMRL & $\begin{array}{l}\text { SEU sensitive depending on operational } \\
\text { conditions; See [Marshall_tns04] }\end{array}$ & RTV & $\mathbf{N} / \mathbf{A}$ & 2 & nsrec04_marshall \\
\hline $\operatorname{sAzZ}$ & IBM & N/A & $\begin{array}{l}\text { SiGe } \\
\text { Jazz }\end{array}$ & JAZZ & HI: (TAMU) PMRL & $\begin{array}{l}\text { SEU sensitive depending on operational } \\
\text { conditions; See [Marshall_tns04] }\end{array}$ & RTV & $\mathbf{N} / \mathbf{A}$ & 2 & nsrecon_marshall \\
\hline
\end{tabular}

Communication Devices

\begin{tabular}{|c|c|c|c|c|c|c|c|c|c|c|}
\hline PE926C31 & Perigrine & $\begin{array}{c}\text { M/A } \\
\text { (Test Chip) }\end{array}$ & $\begin{array}{l}\text { PSSA22 } \\
\text { Driver }\end{array}$ & sos & $\begin{array}{l}\text { P. (IU) SK } \\
\text { P. (UCD) SKAPM }\end{array}$ & $\begin{array}{l}\text { IU: SET } \sigma<2.9 \times 1 \sigma^{-13}(198 \mathrm{MeV}) \\
\text { UDC: SET } \sigma<1 \times 10^{-19}(63 \mathrm{MeV})\end{array}$ & 1 & $5 \mathrm{v}$ & 2 & D031504_RS422 \\
\hline PE926C32 & Peregrine & $\begin{array}{c}\text { N/A } \\
\text { (Test Chip) }\end{array}$ & $\begin{array}{l}\text { RS422 } \\
\text { Receiver }\end{array}$ & $\operatorname{sos}$ & $\begin{array}{l}\text { P: (IU) SK } \\
\text { P. (UCD) SKPM }\end{array}$ & $\begin{array}{l}\text { IU: SET } \sigma<2.9 \times 10^{-13}(198 \mathrm{MeV}) \\
\text { UCD: SET } \sigma<1 \times 10^{-13}(63 \mathrm{MeV})\end{array}$ & 1 & $5 \mathrm{~V}$ & 2 & D031504_RS422 \\
\hline TSSSO1E & ATMEL & $\infty 013$ & 1355 Protocol & CMOS & $\begin{array}{l}\text { H: (TAMU) SB; } \\
\text { Laser: (NPL) SB }\end{array}$ & 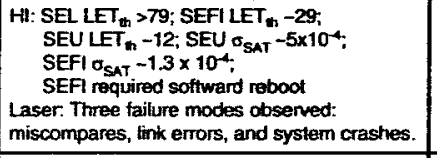 & 2 & $\mathbf{5 v}$ & 1 & $\begin{array}{l}\text { NRL_112103_ } \\
\text { TSS901E; } \\
\text { T091604_TSS901E: } \\
\text { T090203_TSS901E }\end{array}$ \\
\hline UT54LVOS217 & Aeroflex & 0312 & Serializer & CMOS & P: (II) SB & $\begin{aligned} \mathrm{SET} \sigma= & 2 \times 10^{-12} \text { and } 1 \times 10^{-11}(74 \mathrm{MeV}) \\
& 8 \times 10^{-12} \text { and } 3 \times 10^{-11}(198 \mathrm{MeV}) \\
\mathrm{BEF}= & 2 \times 10^{-22} \text { and } 1 \times 10^{-21}(74 \mathrm{MeV}) \\
& 1.02 \times 10^{-21} \text { and } 2.73 \times 10^{-21}(198 \mathrm{MeV})\end{aligned}$ & 3 & $3.3 \mathrm{~V}$ & 2 & $\begin{array}{l}\text { "1102903 } \\
\text { UTS4LVDS217 }\end{array}$ \\
\hline$\$ 2064$ & AMCC & 0303 & Transceiver & CMOS & HI: (TAMU) $N H$ & 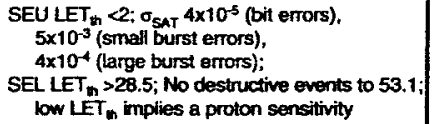 & 3 & $3.3 \mathrm{~V}$ & 2 & T083103_52064 \\
\hline
\end{tabular}

\section{Miscellaneous Devices}

\begin{tabular}{|c|c|c|c|c|c|c|c|c|c|c|}
\hline RHFPPGA & Honeywell & 0314 & FPGA & $\begin{array}{c}0.35 \mathrm{~m} \\
\text { sor }\end{array}$ & $\begin{array}{l}\text { H: (TAMU) TS } \\
\text { P: (IU) TS }\end{array}$ & 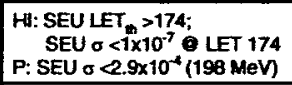 & 1 & $3 v$ & 2 & 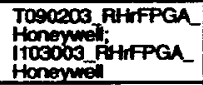 \\
\hline $\mathrm{CO} 1 \mathrm{CD}$ & Teledyne & 0312 & $\begin{array}{l}\text { Solich-State } \\
\text { Retay (OPTO) }\end{array}$ & Hybrid. & $H:$ (TAMU) JH & 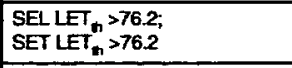 & 1 & $9.5 \mathrm{~V}$ & 1 & TO61800_CD21CD \\
\hline OMR9701 & $\begin{array}{l}\text { Intemational } \\
\text { Rectifier }\end{array}$ & 0313 & $\begin{array}{l}\text { Solid-Stale } \\
\text { Relay (OPTO) }\end{array}$ & Hytrid & HI: (TAMU)SB & SEE LET th $_{n}>29.3$ & 2 & $5 \mathrm{v}$ & 1 & T061303_OMR9701 \\
\hline JANS2N2222A & Microsemi & 0137 & $\begin{array}{l}\text { NPN Silicon } \\
\text { Switcting } \\
\text { Transistor }\end{array}$ & Bipolar & HI: (TAMU) $\mathrm{HH}$ & SET LET $m>53.1$ & 1 & $20 \mathrm{~V}$ & 2 & $\begin{array}{l}\text { T032803_- } \\
\text { JANSEN2022A }\end{array}$ \\
\hline HV583 & SuperTex & NA & Controller & $\begin{array}{l}\text { Microstumer } \\
\text { Array (MSA) }\end{array}$ & $H:(T A M U) S B$ & $\begin{array}{l}\text { SEL LET } \\
\text { SEU }>55.3 \\
\text { SET }\end{array}$ & 2 & $5 / 30 \mathrm{~V}$ & 2 & T022404_HV583 \\
\hline
\end{tabular}

Presented by Martha O'Bryan at NSREC04 Data Workshop, Atlanta, GA, July 22, 2004 
TABLE VIII. SUMMARY OF SEL TEST RESULTS

\begin{tabular}{|c|c|c|c|c|c|c|c|c|c|c|}
\hline $\begin{array}{c}\text { Part } \\
\text { Number }\end{array}$ & Manufacturer & LDC & $\begin{array}{l}\text { Device } \\
\text { Function }\end{array}$ & Process & $\begin{array}{l}\text { Particle: } \\
\text { (Facility,Date) } \\
\text { P.l. }\end{array}$ & $\begin{array}{c}\text { Test Results } \\
\text { LET in MeVocm²hing } \\
\sigma \text { in }^{2}{ }^{2} / \text { device, unless otherwise spocified }\end{array}$ & $\begin{array}{l}\text { SEE } \\
\text { Cat. }\end{array}$ & $\begin{array}{l}\text { Supply } \\
\text { Vottage }\end{array}$ & $\begin{array}{l}\text { Samp. } \\
\text { Size }\end{array}$ & Test Fepont \\
\hline 80387 & Intel & 9809 & $\begin{array}{l}\text { Math } \\
\text { Coprocessor }\end{array}$ & CMOS & Hl: (TAMU) TS & $\begin{array}{l}14.9<\text { SEL LET } \\
\sigma_{\text {SAT }}=1.5 \times 10^{-4}\end{array}$ & $\mathbf{3}$ & $5 \mathrm{~V}$ & 2 & T032603_80387 \\
\hline XA-16 & AMS & Fotght Lot & ASIC & CMOS & HI: (TAMU) JH & $\begin{array}{l}\text { SEI LET } \\
\sigma_{\text {SAI }}-3 \times 10^{-3}\end{array}$ & $3 / 4$ & $\begin{array}{l}+5 V \text { digital } \\
++-5 V \text { analog }\end{array}$ & 4 & T061803_XA16 \\
\hline TC55257 & Toshiba & 0030 & $32 k \times$ B SRAM & CNOS & HI: (TAMU) SB & SEL LET $_{n}>123$ & 1 & $5 V$ & 2 & T032803_TC55257 \\
\hline T36T-GTFE & Agilent & N/A & ASIC & CMOS & HI: (TAMU) SK & $\begin{array}{l}\text { SEL LET }{ }_{n}>51.5 \\
\text { SEL } \sigma<4.0 \times 10^{6} \text { \&ET }=51.5\end{array}$ & 2 & $5 \mathrm{~V}$ & 2 & T022404_GUAST \\
\hline T36T-GTRC & Agitent & NA & ASIC & CMOS & HI: (TAMU) SK & 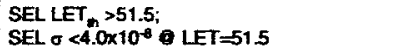 & 2 & $5 \mathrm{~V}$ & 2 & T022404_GLAST \\
\hline AD7675 & Analog Devices & $\begin{array}{l}\text { No LDC } \\
\text { (Test Chip) }\end{array}$ & $A D C$ & CMOS & HI: (TAMU) RLTS & SEL LET $<8.6$ & 4 & $5 \mathrm{~V}$ & 2 & T1111603_AD7675 \\
\hline ADS8323 & Texas hotrument & No Loc & $A D C$ & CMOS & H: (TAMU) AL/TS & $S E I L E T_{n}-17.2$ & 4 & $5 \mathrm{~V}$ & 2 & T111603_ADseses \\
\hline
\end{tabular}

\section{FEATURED TeSt Results and Dicussion}

As in our past workshop compendia of GSFC test results, each DUT has a detailed test report available online at $\mathrm{http} / / /$ radhome.gsfc.nasa.gov [8] describing in further detail test method, SEE conditions/parameters, test results, and graphs of data. This section contains a summary of testing performed on a selection of featured parts.

\section{A. Si-Nanocrylstal NVMs}

Shown in Figure 1 are the bit errors in a $4 M$ nanocrystal nonvolatile memory for exposures of $1 \times 10^{7}$ particles $/ \mathrm{cm}^{2}$. The memories were produced by Freescale (Motorola), as part of their $90 \mathrm{~nm}$ technology development. Both static and dynamic $(10 \mathrm{kHz})$ read testing were performed, but all the errors appeared to be static errors, corresponding to electrons lost off the nanocrystal storage element. No errors were observed which could be attributed to the control circuits. These memories are normally programmed by channel hot electron injection and erased by Fowler-Nordheim tunneling, much like floating gate nonvolatile memories. Further testing of the write and erase modes is planned. [T022204_Motorola]

\section{B. $A M C C S 2064$}

The AMCC S2064 1.3 GHz Quad Serial Transceiver was monitored for latchup induced high power supply currents and data disruptions and errors by exposing it to a number of heavy ion beams at the Texas A\&M University Cyclotron Single Event Effects Test Facility.

Difficulties were encountered during the de-lidded process. The DUT die was encapsulate in two plastics, an outer plastic covers the inner plastic and the top arc of the bond wires. This outer plastic was etched by fuming nitric acid. The inner plastic was insensitive to this acid and needed to be removed by an application of sulfuric acid. During the first effort to de-lid the devices where complete exposure of the die was the goal, the de-lidding process appeared to proceed normally. Initial inspection of the uncovered die appeared normal. However, when the de-lidded devices were taken to the facility, all the bond wires had lifted from the die surface, yielding nonfunctional devices. The second de-lidding process was to remove as much of the second encapsulant without exposing the bond wires. As this was a very sensitive process, the two devices that were successfully de-lidded had slightly different overlayer thicknesses (12 and 7 mils, for DUTs 1 and 2 respectively).

The test configuration utilized a $3.6 \mathrm{GHz}$ Bit Error Rate Tester (BERT) with generator and detector/analyzer. The DUT was mounted on a commercial board specifically designed for radiation testing of this part. The test setup was wired for either one pass through the DUT or four passes. For further details on the test set-up see the test report "Single Event Effects (SEE) Testing of the AMCC S2064 1.3 GHz Quad Serial Transceiver". [ref: T083103_S2064]

The Xenon particles did not have sufficient range to penetrate into the sensitive regions after passing through the inner encapsulant layer. Therefore, the Xenon beam could not give a sufficient test for latchup. The highest LET beam available with sufficient penetration range was Krypton, but only for DUT 2 with the 7 mil overlayer. After being exposed to $1 \times 10^{7} \mathrm{Kr}$ ions $/ \mathrm{cm}^{2}$, no latchup events were observed on the 7 mil DUT, therefore, the latchup threshold for the S2064 devices is greater than $28.5 \mathrm{MeV} \cdot \mathrm{cm}^{2} / \mathrm{mg}$.

The simplest method for processing the data is to determine the total number of errors produced in a given run and determine the upset cross-section from the total ion fluence for that run. However, if any events occurred that produced more than one error for a given ion, then this method would misrepresent the upset rate. These multiple bit events, or burst errors were seen during testing of the AMCC S2064. To account for burst errors, a definition of when a burst error starts and stops was defined. We defined this condition to be two control characters ( 20 bits) being passed correctly. That is, if a second bit error is encountered before 20 bits have transferred correctly, then a burst error has occurred. The burst length (how long the burst error condition lasts in bits, erroneous and valid bits combined) is defined by the start bit as above and the bit that starts 20 correct bits (i.e., two correct control characters were passed). Post-processing the data in this fashion yields single bit errors and burst errors (each burst error has an associated burst length).

A histogram of the burst length was viewed on the BERT and there was a double peak structure noted. Burst lengths ranged from 2 bits up to about 600 - 700 bits, then there were no burst lengths until greater than 6000 bits. It was determined that this second peak in the histogram was from burst events 
that were lasting so long that the BERT initiated a RESYNC event and recovered normal operation after the RESYNC. All burst events that lasted less than 700 bits returned to normal operation without any intervention by the BERT system. Therefore, the burst errors in this analysis were split into two categories - ones that recovered with no intervention and ones that required intervention to resume normal operations. These are referred to as small and large burst events, respectively.

Figure 2 shows the cross-section versus LET curve for the single bit errors. There are three results of interest for the single bit error case. First the single bit error upset mode was more than an order of magnitude lower in cross-section than for burst errors. Second, there is little difference between the one-pass and four-pass cases. This implies, at least for the statistic levels of these observations, that the single bit errors are not produced in the data transmitting/receiving stages that are quadrupled in the four-pass case. Third, the errors were produced by all ion beams used, even the Neon beam. This means the LETth to produce single bit errors is $>2 \mathrm{MeV} \cdot \mathrm{cm}^{2} / \mathrm{mg}$, implying that protons are likely to produce these events (via spallation reactions and possibly via direct ionization). No proton testing was done to investigate this possibility but is highly recommended.

Figure 3 and Figure 4 show the cross-section for small and large burst errors as a function of the LET. Comparing the saturation cross-section levels of these curves with that for the bit errors, the more than an order of magnitude difference is easily seen. The small burst error events are the most likely events, followed by the large burst errors, and finally by the single bit errors.

Unlike the single bit error case, the burst errors do seem to be sensitive to the number of passes through the DUT. While the exact factor of four is not seen, Figure 3 and Figure $\mathbf{4}$ do show that the four-pass case is significantly larger than the single-pass case. This implies that the data transmission/receiving section of the device is the main area sensitive to producing burst-type errors.

In summary, all three types of errors observed were seen at the lowest test LET $\left(2 \mathrm{MeV} \cdot \mathrm{cm}^{2} / \mathrm{mg}\right)$, yielding threshold LETs of less than two. Saturation cross-sections for the three error modes were $4 \times 10^{-5} \mathrm{~cm}^{2}$ (bit errors), $5 \times 10^{-3} \mathrm{~cm}^{2}$ (small burst errors), $4 \times 10^{-4} \mathrm{~cm}^{2}$ (large burst errors).

The AMCC S2064 1.3 GHz Quad Serial Transceivers are considered category 3 devices. While no destructive events were observed to the highest LET able to penetrate the overlayer (approximately $28 \mathrm{MeV} \cdot \mathrm{cm}^{2} / \mathrm{mg}$ ), the upset rate and modes may require substantial mitigation to achieve successful operation. Additionally, the low threshold LET implies a proton sensitivity that was not investigated. Space-borne rates could be substantially higher than predicted with heavy ions alone if proton sensitivity is shown, especially if the devices are sensitive to direct proton ionization.

\section{Honeywell RHrFPGA}

Heavy Ion testing was performed at Texas A\&M University Radiation Effects Facility on the Honeywell RadiationHardened re-programmable Field-Programmable Gate Array
(RHrFPGA). Testing characterized the RHrFPGA Single Event Upset (SEU) sensitivity to verify compliance with its Soft Error Rate (SER) radiation design requirements. The test evaluated the FPGA using eight different test programs and configurations. Seven were optimized for SEU testing to evaluate specific internal memory elements within the FPGA, and one test program represented a current RHrFPGA application. The RHrFPGA test devices did not experience SEU or other SEE to the maximum available test LET of $174 \mathrm{MeV} \cdot \mathrm{cm}^{2} / \mathrm{mg}$ at minimum rated supply voltage $(3.0 \mathrm{~V})$; This result applied to all eight tests for fluences of $1.0 \times 10^{7}$ ions $/ \mathrm{cm}^{2}$ per test.

Proton testing was performed at Indiana University Cyclotron Facility. Testing characterized RHrFPGA sensitivity to proton-induced SEU. The test included six different test programs and FPGA configurations, which were optimized to evaluate the RHrFPGA's two unique types of memory elements. The RHrFPGA test device was irradiated to a proton fluence of $3.4 \times 10^{13} \mathrm{p} / \mathrm{cm}^{2}$ at $203 \mathrm{MeV}$ beam energy, corresponding to $2.0 \mathrm{Mrad}(\mathrm{Si})$ total dose per device. The test parts did not exhibit SEU or any other SEE, demonstrating that the RHrFPGA is essentially immune to proton-induced SEU. [T090203 RHrFPGA Honeywell] [1103003_RHrFPGA_Honeywell]

\section{SUMMARY}

We have presented recent data from SEE on a variety of mainly commercial devices. It is the authors' recommendation that this data be used with caution. We also highly recommend that lot testing be performed on any suspect or commercial device.

\section{ACKNOWLEDGMENT}

The Authors would like to acknowledge the sponsors of this effort: NASA Electronic Parts and Packaging Program (NEPP), NASA Flight Projects, and the Defense Threat Reduction Agency (DTRA) under IACRO 03-40351 and 04-40641.

\section{REFERENCES}

[1] Donna Cochran, et al. "Current Total Ionizing Dose Results and Displacement Damage Results for Candidate Spacecraft Electronics for NASA," submitted for publication in IEEE NSREC04 Data Workshop, July 2003.

[2] B. Hyman, "Texas A\&M University Cyclotron Institute, K500 Superconducting Cyclotron Facility," http $/ /$ cyclotron.tamu.edu/ facilities.htm, July 2003.

[3] National Superconduction Cyclotron Laboratory (NSCL) at Michigan State University, http:/www.nscl.msu.edu/, May 2004.

[4] C. M. Castaneda, "Crocker Nuclear Laboratory (CNL) Radiation Effects Measurement and Test Facility," IEEE NSRECO1 Data Workshop, pp. 77-81, July 2001 .

[5] C. C. Foster, S. L. Casey, P. Miesle, N. Sifri, A. H. Skees, K. M. Murray, "Opportunities for Single Event and Other Radiation Effects Testing and Research at the Indiana University Cyclotron Facility," IEEE NSREC96 Data Workshop, pp. 84-87, July 1996.

[6] J. S. Melinger, S. Buchner, D. McMorrow, T. R. Weatherford, A. B Campbell, adn H. Eisen, "Critical evaluation of the pulsed laser method for single event effects testing and fundamental studies," IEEE Trans. Nucl. Sci., vol 41, pp. 2574-2584, Dec 1994.

Presented by Martha O'Bryan at NSREC04 Data Workshop, Atlanta, GA, July 22, 2004 Page 7 of 8 
[7] D. McMorrow, J. S. Melinger, and S. Buchner, "Application of a Pulsed Laser for Evaluation and Optimization of SEU-Hard Designs," IEEE Trans. Nucl. Sci., vol 47, no. 3, pp. 559-565, June 2000.

[8] NASA/GSFC Radiation Effects and Analysis home page, http://radhome.gsfc.nasa.gov

[9] [T083103_S2064.pdf] J. Howard, M. Carts, and T. Irwin, "Single Event Effects (SEE) Testing of the AMCC S2064 $1.3 \mathrm{GHz}$ Quad Serial Transceiver," T083103_S2064.pdf, Aug 2003.

[10] [T090203_RHrFPGA_Honeywell] J. Lintz, "Single Event Effects Radiation Test Report for the RHrFPGA Radiation-Hardened FieldProgrammable Gate Array," T090203_RHrFPGA_Honeywell, Sept 2003.

[11] [I103003_RHrFPGA_Honeywell] J. Lintz, "Proton Single Event Effects Radiation Test Report for the RHrFPGA Radiation-Hardened FieldProgrammable Gate Array," I103003_RHrFPGA_Honeywell, Oct 2003.

[12] [T022204_Motorola] T. Oldhaml, H. Kim, and S. Moharmmed, "Heavy Ion Testing of the Motorola Nano-crystal Nonvolatile Memory," T022204_Motorola, Feb 2004.

[13] [nsrec04_MSU] R. Ladbury, et al., "Performance of the High-Energy Single-Event Effects Test Facility (SEETF) at Michigan State University's National Superconducting Cyclotron Laboratory (NSCL)," submitted for publication to 2004 IEEE Trans. on Nucl. Sci., Dec 2004.
[14] [nsrec04_Marshall] Paul Marshall, et al., "A Comparative Study of Heavy Ion and Proton Induced Bit Error Sensitivity and Complex Burst Error Modes in Cormmercially Available High Speed SiGe BiCMOS," submitted for publication to 2004 IEEE Trans. on Nucl. Sci., Dec 2004.

[15] [T022204_Motorola] Timothy Oldham, et al., "Heavy lon Testing of the Motorola Nano-crystal Nonvolatile Memory," http://radhome. gsfc.nasa.gov/radhome/papers/T022204_Motorola.pdf, Feb. 2004.

[16] [T083103_S2064] Jim Howard, et al., "Single Event Effects (SEE) Testing of the AMCC S2064 1.3 GHz Quad Serial Transceiver," http://radhome.gsfc.nasa.gov/radhome/papers/T083103_S2064, Aug. 2003.

[17] [T090203_RHrFPGA_Honeywell] John Lintz, "Single Event Effects Radiation Test Report for the RHrFPGA Radiation-Hardened FieldProgrammable Gate Array," http:/tradhome.gsfc.nasa.gov/radhome/ papers/T090203_RHrFPGA_Honeywell.pdf, Sept. 2003.

[18] [i103003 RHrFPGA Honeywell] John Lintz, 'Proton Single Event Effects Radiation Test Report for the RHrFPGA Radiation-Hardened Field-Programmable Gate Array," http:/radhome.gsfc_nasa.gov/ radhome/papers/i103003_RHrFPGA_Honeywell.pdf, Oct. 2003. 